\title{
An Analysis of CNS Tumors in Squash Preparations with Histological Correlation
}

\author{
Dr.Teleflo Boopathy ${ }^{1}$, Dr. V.Karthika ${ }^{2}$ \\ ${ }^{I}$ (Assistant Professor, DepartmentOf Pathology. GovernmentkilpaukMedical College, India) \\ ${ }_{2}^{2}$ (Assistant Professor, Department Of Pathology. GovernmentkilpaukMedical College, India)
}

\begin{abstract}
:
Background: Squash cytologyis now a well establishedand universally accepted techniquein diagnosing a wide range of CNS lesions and is currently employed for both therapeutic and prognostic reasons.

Aims and Objectives: This study was conducted with an aim to correlate squash smears with histopathology and to compare statistical data employing sensitivity, specificity and diagnostic accuracy of squash cytology. The study also aims to find the pitfalls and limitations of Squash technique.

Materials and Methods: An analysis of 100 brain tumour was done by squash preparation with histopathological correlationto evaluate the usefulness of the procedure. Crush Cytology smears were stained by Rapid H\&E. Histopathology smears were made from formalin fixed tissues sent separately and stained with $H \& E$.

Results: Out of 100 cases, complete correlation with the final diagnosis was achieved in $82 \%$. Diagnostic accuracy increased to $92 \%$ when cases of partial correlation due tograding deviations were included. The sensitivity and specificity of neuroepithelialtumours were $86.6 \%$ and $98.18 \%$ respectively. The positive predictive value was $97.5 \%$ and negative predictive value was $90 \% .10 \%$ partial correlation defect was not significant to affect the management of patient.

Conclusion: The study shows it is possible to make correct diagnosis by squash preparation in $92 \%$ of the cases. The diagnostic yield is very high when the technique is combined withhistopathology. Itmaybe used as auseful adjunct to conventional histopathology.
\end{abstract}

Keywords: CNS, histopathology, crush cytology.

\section{Introduction}

Intraoperative consultation is an important component in the surgical management of brain tumours. Critical decision regarding treatment and extent of surgical aggression can sometimes depend on an appropriate intraoperative cytodiagnosis. At the operating table, many a time theneurosurgeon wants to know if the biopsy is from a representative site. The pathologist has to bear all this in mind andmust be able to arrive at a diagnosis while the patient is on the operating table. The procedure adopted to achieve all these parameters must be simple, easy to perform, rapid and accurate. Crush cytology could achieve most of these requirements. The capability of diagnosing a lesion from a small tissue within a brief period of time is difficult with other methods. In experienced hands, the smear technique attains a high degree of accuracy. But errors do occur and in such cases decision should always be made on the basis of $\mathrm{H} \& \mathrm{E}$ stained paraffin sections which is the gold standard.

\section{Materials and methods:}

Biopsy samples collected from Dept of Neurosurgery were studied over a period of 30 months. A total of 100 cases were taken. Tissue biopsy of all intracranial space occupying lesion were obtained during surgery in the theatre moistened with saline.

\section{Preparation of Crush Smear}

2 or more tissue fragments usually measuring no more than $2 \mathrm{mmin}$ diameter were first examined with the help of a magnifier to see if the specimen appeared necrotic or haemorrhagic. The apparently viable tissue was then placed on the centre of a labelled glass slide. A second labelled slide was placed over the first slide on top of the tissue fragment. Then sufficient pressure was applied between the tips of the thumb and index finger to spread the tissue. If the tissue was soft it spreads easily. If it was hard and firm it resisted spreading. In such cases smaller tissue fragments were used to prepare the smear.The two slides were thenpulled apart to produce two thin well prepared smears. One smear was immediately fixed in 95\% ethyl alcohol for 1- 2 minutes and stained byrapid H \& E method. Remaining tissue was fixed in 10\% neutral formalin, paraffin blocks prepared and stained with $\mathrm{H} \& \mathrm{E}$ stain. 


\section{Results}

Table 1 shows incidence of CNS lesions in present study. Complete correlation with the final diagnosis was achieved in $82 \%$ of cases. Diagnostic accuracy increased to $92 \%$ in cases of partial correlation mainly due to grading deviations. $5 \%$ of cases had wrong diagnosis. 4 cases showed sampling error. The discrepancies obtained on comparing squash with Histopathological diagnosis is depicted in Table 2."Fig 1" showed the correlation between disease and diagnosis.

Table 1: Incidence of brain tumours in present study

\begin{tabular}{|l|l|}
\hline Disease & Incidence \\
\hline Abscess & 2 \\
\hline Astrocytoma & 33 \\
\hline AV Malformation & 1 \\
\hline Cavernoma & 2 \\
\hline Craniopharyngiomas & 1 \\
\hline DesmoplasticNeuroblastoma & 1 \\
\hline Ependymoma & 2 \\
\hline Epidermal cyst & 2 \\
\hline Hemangiopericytoma & 1 \\
\hline Lipoma & 1 \\
\hline Medulloblastoma & 3 \\
\hline Meningioma & 15 \\
\hline Metastatic Carcinomatous Deposit & 2 \\
\hline Mixed Glioma & 3 \\
\hline Oligodendroglioma & 2 \\
\hline Pituitary Adenoma & 2 \\
\hline PNET & 1 \\
\hline Schwannoma & 16 \\
\hline Tuberculoma & 8 \\
\hline Vascular Neurofibroma & 1 \\
\hline &
\end{tabular}

Table 2: Discrepancies noted when comparing squash cytology with histopathological diagnosis

\begin{tabular}{|l|l|}
\hline Crush Diagnosis & Final Histopathological Diagnosis \\
\hline Lymphoma & Grade IV Astrocytoma \\
\hline Meningioma - Fibroblastic & Pilocytic Astrocytoma \\
\hline Sub Ependymoma & Astrocytoma Grade IV \\
\hline Meningioma & $\begin{array}{l}\text { Mixed Glioma (Ependymoma with } \\
\text { Astrocytoma }\end{array}$ \\
\hline Grade IV Astrocytoma & Metastatic Carcinomous Deposit \\
\hline
\end{tabular}

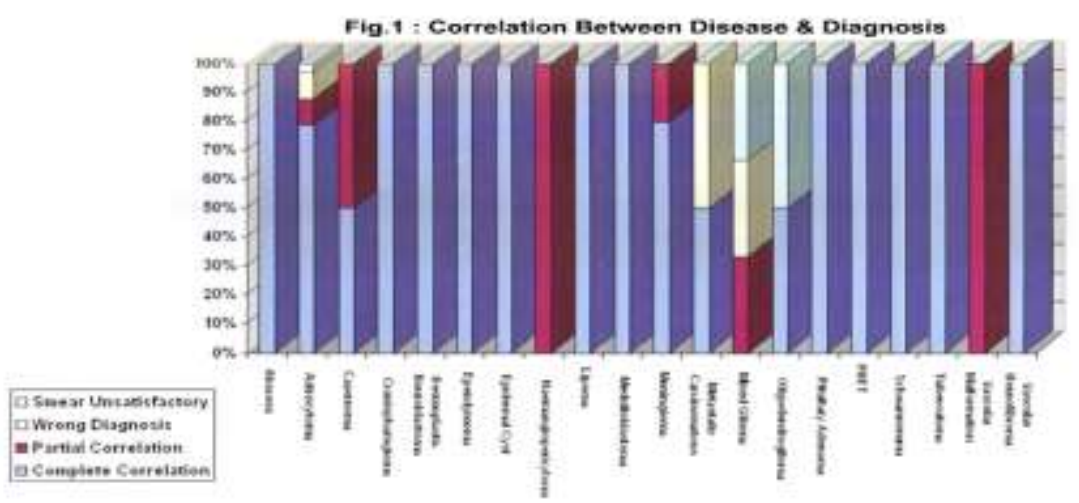

\section{Discussion}

Tumours of the brain have unique characteristics that set them apart from neoplastic processes elsewhere in the body. Even though they amount toless than $2 \%$ of all malignant neoplasms their increased incidence in recent years has created great interest in studying them. The main aim of the pathologist is to give the surgeon a quick and accurate diagnosis as early as possible. Surgery for lesions on the brain is time consuming. Opening and closing of the skull frequently is also difficult. Many a time the neurosurgeon wants to know if the tissue taken is from a representative site. The procedure adopted to achieve all these parameters must be simple, easy to perform, rapid and accurate. Crush cytology is able to meet most of these requirements.

The advantages of squash/crush cytology in comparison over frozen section are that it does not need any expensive and sophisticated equipment like Cryostat. Cytological details are also more accurate. [1] 
A comparative correlation between crush cytology and histopathology is highlighted in Table3[2,3,4]. Out of 100 cases studied complete correlation was achieved in 82\% cases. Diagnostic accuracy increased to $92 \%$ when cases of partial correlation mainly due to grading deviations were included. The sensitivity and specificity of Neuroepithelial lesions were $86.67 \%$ and $98.18 \%$ respectively. The positive predictive value was $97.5 \%$ and the negative predictive value was $90 \%$.

Table 3:ComparitiveCorelation between Crush Cytology and Histopathologic - A comparative study

\begin{tabular}{|l|l|}
\hline Name of the study & Complete Correlation in percentage \\
\hline Tigner et al & $81.3 \%$ \\
\hline Patty et al & $87 \%$ \\
\hline Shaw et al & $89.7 \%$ \\
\hline Present Study & $82 \%$ \\
\hline
\end{tabular}

In the present study there were 4 cases of missed diagnosis due to poor smear technique. A case of Malignant Oligodendroglioma was missed because of drying artefact and degenerative changes. A case Of Schwannoma was missed because the tissue was firm and resisted spreading making the material inadequate. The material obtained was too necrotic to give conclusive opinion in a case of Mixed Glioma and Anaplastic Astrocytoma.

$10 \%$ cases showed only partial correlation. The grading of 3 casesof Astrocytoma was incorrect. 4 cases of Meningioma the histological typing varied. Sampling error was observed in a case of Oligoastrocytoma were the Astrocytic component was not present in the tissue crushed. This proves that gradingof CNS tumours not very helpful in crush preparations. [4] The sample of the tumour may not be representative of the whole lesion and sometimes thegrading was a level lower or higher thanthefinal histopathological diagnosis. The material was too necrotic to give conclusive opinion on two cases of Mixed Glioma and Anaplastic Astrocytoma.

33 cases of Astrocytoma "fig2" were diagnosed by crush cytology. One important finding in all Astrocytomas were that the cells were attached to their vessels walls by their long glial fibres and foot processes.[5] A case of Astrocytoma Grade 4 was diagnosed as Lymphoma on crush preparation because the round lymphoid cells admixed with reactive astrocytes caused interpretational difficulties. A case of Astrocytoma Grade4 was misdiagnosed as Subependymoma on crush because in both the tumour cells can attach to blood vessels.[6]
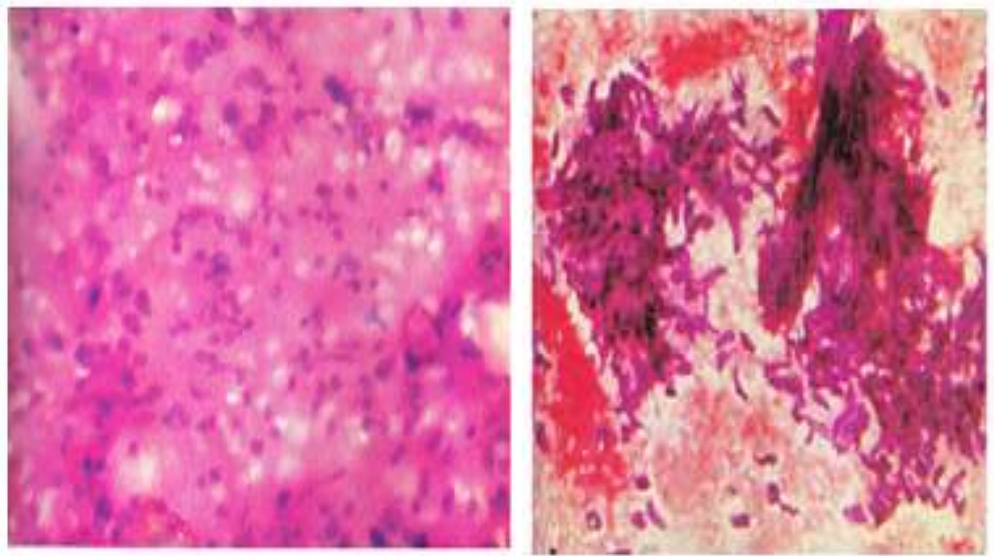

Fig2: Low grade Astrocytoma showing tumour cells close to blood vessels. Nuclei are oval and cytoplasm is scanty 200x (H\&E).

Fig3:Schwannoma: Smear shows spindle shaped neoplastic cells arranged in discrete groups in aneosinophilic and haemorrhagic background. 200x (H\&E) 

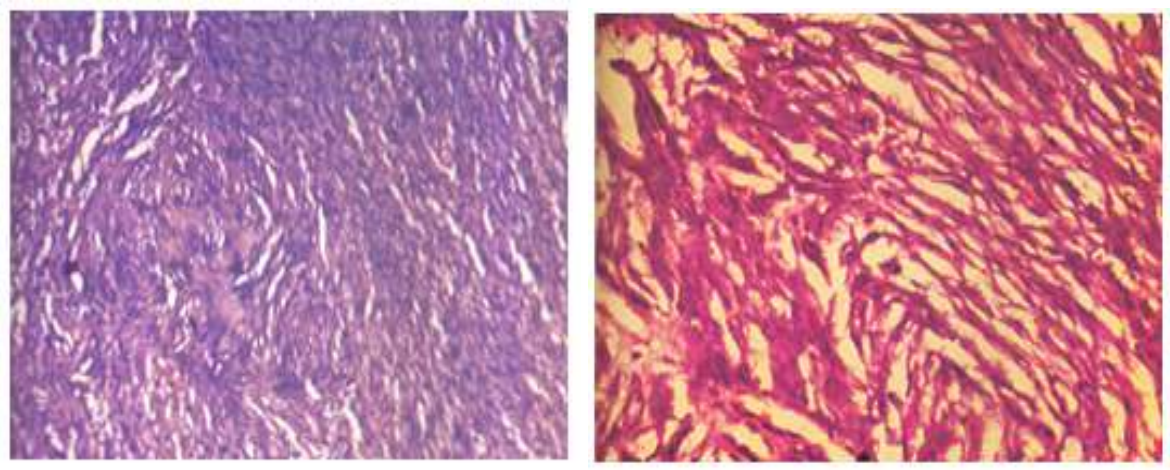

Fig4: Schwannoma showing Antoni A and Antoni B area. 50X (H\&E)

The next large group was Schwannoma "fig3" "fig4" followed by Meningioma. As in one of our case difficulties in interpreting Fibroblastic Meningioma "fig5" from Astrocytoma can occur because of the fibrillary processes. In such cases focal areas would still show presence of whorls and arachnoidal cells which would help to clinch the diagnosis.[7] It is easy to diagnose Psammomatous Meningioma because the calcific bodies cause grittiness while smearing. Other features should also be looked into because Pilocytic Astrocytoma "fig6" and Oligodendroglioma canalso cause calcific bodies. A case diagnosed as Atypical Meningioma on crush turned out tobe Meningothelial Meningioma. Cells at the periphery may artifactually appear bigger.

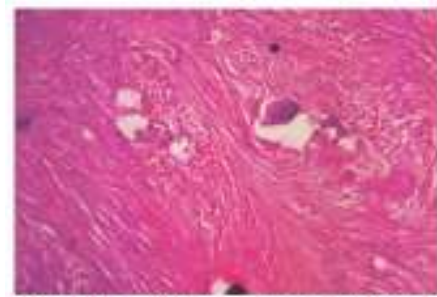

Fig6:PilocyticAstrocytoma xhibiting biphasic pattern withcompact bipolar cells and cystic areas with calcification, $50 \mathrm{x}(\mathrm{H} \& \mathrm{E})$.

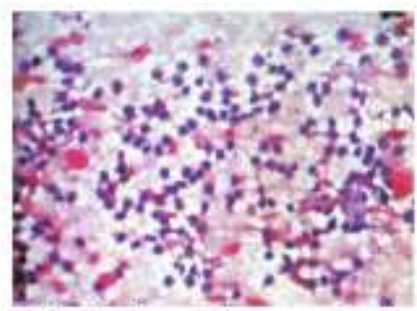

Fig8: Pituitary Adenoma showing cells of varying sizes in a eosinophilic background. $200 \mathrm{X}$ (H\&E)

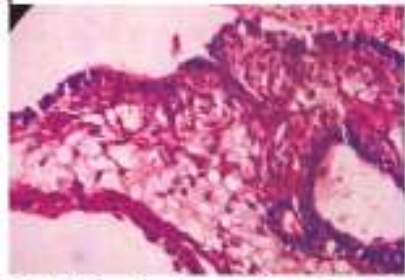

Fig10: Craniopharyngioma showing anastomosing epithelial islands with peripheral palisading of nuclei and a central loose stellate reticulum. 200X (H\&E)

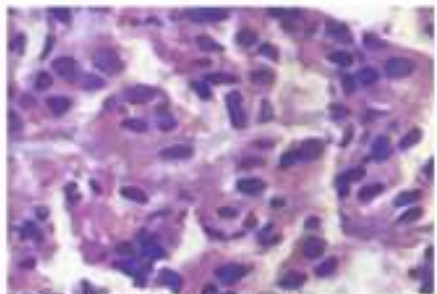

Fig7; Pituitary Adenoma showing pleomorphic cells with abundant eosinophiliccytoplasm. Considerable variation in size of cells seen, $200 \mathrm{x}$ (H\&E)

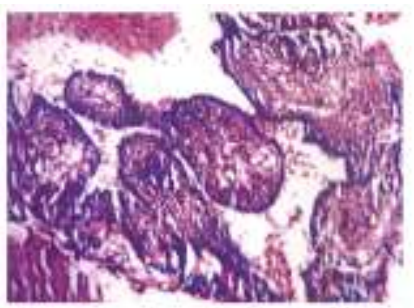

Fig9: Craniopharyngioma showing anastomosing epithelial islands with peripheral palisading of nuclei and a central loose stellate reticulum. $50 \mathrm{X}$ (H\&E)

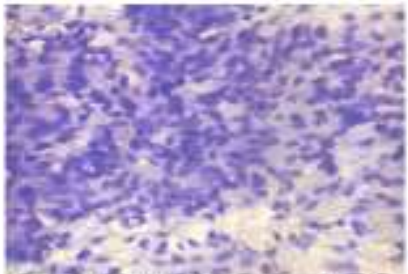

Fig11: Medulloblastoma showing closely packed tumour cells with elongated carrot shaped nucleus and forming rossettes. 200X (H\&E) 


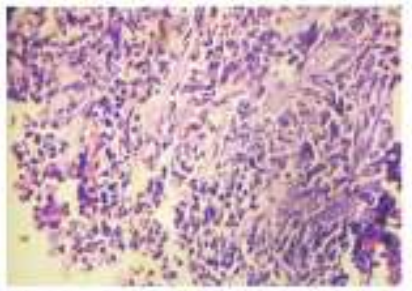

Fig12: Medulloblastoma showing closely packed tumour cells and Homer Wright Rosettes. 200X (H\&E)

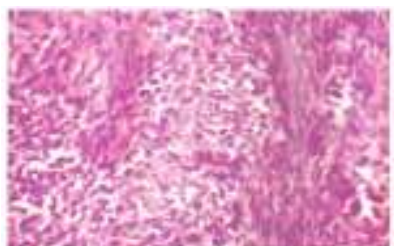

Fig14: Desmoplastic Neuroblastoma showing

Micronodular zones of reduced cellularity (pale islands) seen. $200 \mathrm{X}$ (H\&E)

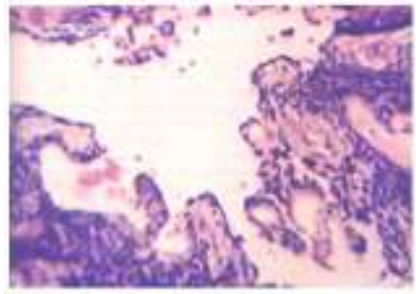

Fig16: Ependymoma showing neoplastic cells which are round to oval with perivascular pseudo Rosettes. 50X (H\&E)

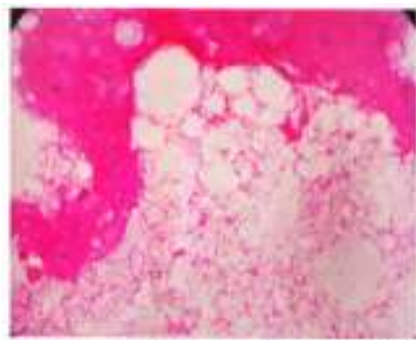

Fig18: Lipoma showing mature adipocytes with glial tissue. $50 \mathrm{X}$ (H\&E)

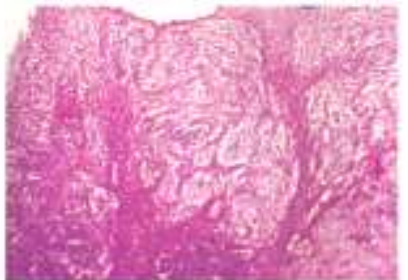

Fig13:

Desmoplastic

Neuroblastoma. Micronodular zones of reduced cellularity (pale islands) seen. $50 \mathrm{X}$ (H\&E)

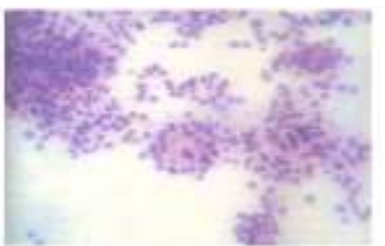

Fig15: Ependymoma: Cells smear out individually and have an epithelial appearance. Numerous Rosettes are also seen. 50X (H\&E)

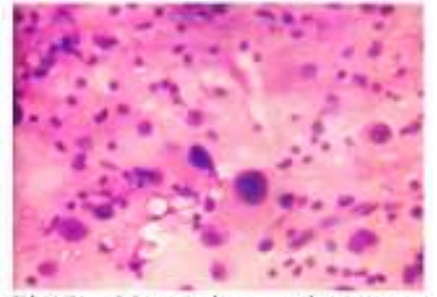

Fig17: Metastatic carcinomatous deposit showing tumour cells with eosinophilic cytoplasm and bizarre hperchromatic nucleus. $200 \mathrm{X}$ (H\&E)

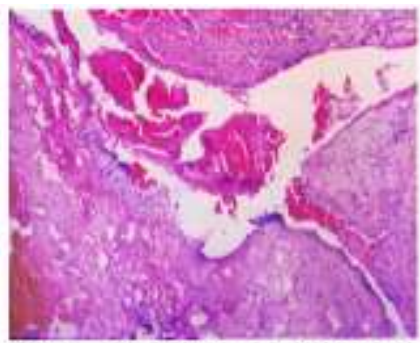

Fig19: Cavernous Haemangioma showing enlarged vessels filled with haemonhage. $200 \mathrm{X}$ (H\&E)

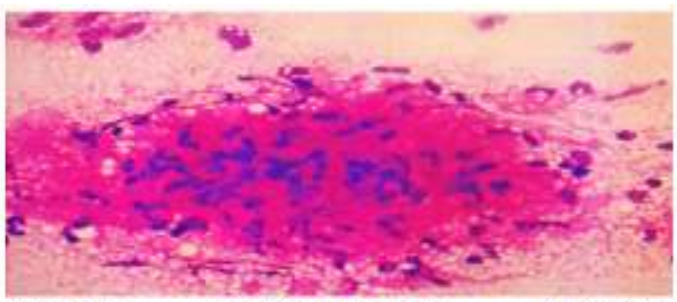

Fig20: tuberculoma showing epitheloid cells and lymphocytes in a necrotic background, $200 X$ (H\&E) 
All cases of Pitutary Adenoma "fig7" "fig8",Craniopharyngioma "fig9 fig10", Medulloblastoma "fig11" "fig12" , DesmoplasticNeuroblastoma"fig13" "fig14"and Ependymoma "fig 15" "fig16" were diagnosed rightly.Age of the patient, clinical and CT findings helped in arriving at the right diagnosis. [8]The tissue was soft and smeared easily in Pituitary Adenoma. In Craniopharngiomaon squashing it did not smear well, appeared membranous and revealed basaloid cells topped by squamous cells.In Ependymoma the cells were closely attached to the vessels producing perivascular pseudorosettes.

2 cases of Metastatic carcinoma"fig17" were diagnosed. The tendency of the cells to separate from vessel wall clearly distinguished it from Astrocytoma.[9]. VasularNeurofibroma, Lipoma "fig18", Cerebral Abscess, Epidermoid cyst and Cavernous Haemangioma "fig19" diagnosed on squash correlated accurately with histopathology.

8 cases of Tuberculomas "fig20" were diagnosed on crush and confirmed by histopathology. One case was diagnosed as Tuberculoma on crush preparation. CT also confirmed thediagnosis but when the remaining tissue was processed for paraffin section it showed only normal brain tissue. The abnormal tissue might have been utilised for smear preparation. In such cases Crush cytology is complementary to histopathology in arriving at a diagnosis.

As there were no cases of haemangioblastoma, Myeloma, Chordoma, PinealTumours and Tumours of the choroid plexus the efficiency of squash preparationcould not be commented inthesecases.However other authors have exemplified the usefulness of squash preparation in these tumours too.[10]

\section{Conclusion}

Of the total 100 cases complete correlation with the final diagnosis was achieved in $82 \%$ of the cases.Diagnostic accuracy was increased to $92 \%$ when cases of partial correlation mainly due to grading deviations were included. The sensitivity and specificity of Neuroepithelialtumours were $86.67 \%$ and $98.18 \%$ respectively. The Positive predictive value was $97.5 \%$ and the Negative predictive value was $90 \% .10 \%$ partial correlation defectwas not significant enough to affect the management of patient. Correlation withCT and clinical details were helpful in improving the accuracy rate. The problems encountered were improper squash technique, sampling error leading to Grading discrepancy and inadequate diagnosis in cases of mixed lesions.

To conclude Crush cytology is a valuable, simple, easily reproducible, cost effective and clinically significant procedure. It could be used as an intraoperative diagnostic tool to assist the surgeon for preoperative treatment plan.

\section{References}

[1]. BeuvonF ., Varlet P. Fallet- Bianloc, Daumas - Duport C. The "Smear" technique for the extemporaneous examination: diagnostic contribution to neurosurgical pathology 1 :AnnPathol. 2000 Oct ; 20(5) : 499-506.

[2]. Tilgner J, Herr M, Ostertag C, Volk B. Validation of intraoperative diagnosis using smear preparations from stereotactic brain biopsies: Introoperative versus finaldiagnosis - influence of clinical factors. Neurosurgery. 2005: 56:257-62.

[3]. Patty I. Central nervous system tumors: A clinicopathological study. J Dohuk Uni. 2008: 11: 173-175.

[4]. Priyanka Chand, Sonal Amit, Errors, limitations, and pitfalls in the diagnosi of central and peripheral nervous system lesions in intraoperative cytology and frozen sections.Journal of cytology,

[5]. Vol. 33, No. 2, April - June 2016, pp93-97.

[6]. Suzanne Z. Powell (2005) Intraoperative consultation, Cytological Preparations, and Frozen Section in the Central Nervous System. Archives of Pathology \& Laboratory Medicine: December 2005. Vol 129, No. 12, pp. 1635 - 1652.

[7]. Adekumle M. Adesina Intraoperative consultation in the diagnosis of Paediatric Brain tumors. Archives of Pathology \& Laboratory Medicine. 2005 Vol 129. No 12pp 1653-1660.

[8]. Mitra S, Mohan K, Sharma V, Mukhopadhya D. Squash preparation: A reliable diagnostic tool in the intraoperative diagnosis of central nervous system tumors. J Cytol2010;27;81-5.

[9]. Daniel J Brat, Richard Prayson Diagnosis of malignant glioma: Role of neuropathology. Journal of Neuro- Oncology89(3) :287311 Oct 2008 .

[10]. Yuji Uematsu,YoshihiroOwai The usefulness and problem of intraoperative rapid diagnosis in surgical neuropathology. Brain Tumor Pathology 24(2):47 - 52. February 2007.

[11]. Stephen G. Silverberg Principles and Practice of Surgical Pathology and Cytopathology third edition Vol 3 1997 62 2905-3001.s 